\title{
A549 cell proliferation inhibited by RNAi mediated silencing of the Nrf2 gene ${ }^{1}$
}

\author{
Bo Zhang ${ }^{\mathrm{a}, \mathrm{b}}$, Chen Xie ${ }^{\mathrm{a}}$, Jing Zhong ${ }^{\mathrm{a}}$, Hanyin Chen ${ }^{\mathrm{a}, \mathrm{b}}$, Hongying Zhang ${ }^{\mathrm{a}}$ and \\ Xiaoqin Wang ${ }^{\text {a,* }}$ \\ ${ }^{a}$ Key Laboratory of Xinjiang Endemic Phytomedicine Resources, Ministry of Education, Shihezi \\ 832002, China \\ ${ }^{b}$ Pharmacology Department, School of Pharmacy, Shihezi University, Shihezi 832002, China
}

\begin{abstract}
Non-small-cell lung cancer (NSCLC), the most common type of lung cancers, is resistant to initial chemotherapy intrinsically. The expressions of xenobiotic metabolism genes, antioxidants, and drug efflux proteins are increased in NSCLC. In addition, a redox-sensitive transcription factor named $\mathrm{Nrf} 2$ regulates the drug resistance via the expression of electrophile, oxidants detoxification enzymes and efflux mechanism. As was detected by real-time PCR, inhibiting Nrf2 expression through the transfection of shRNA plasmids in A549 cells significantly inhibits the expressions of glutathione pathway genes, antioxidants and multidrug resistance proteins. Using biochemical assays and free radical medical experiments in vitro, it was identified that the RNAi-mediated reduction of Nrf2 expression in lung cancer cells induces the generation of reactive oxygen species, decreases the level of reduced glutathione and results in an increase in the A549 cell proliferation inhibition rate. Thus, targeting Nrf2 activity in NSCLC could be a practical way to inhibit tumor growth and eliminate chemoresistance.
\end{abstract}

Keywords: A549 cell, cell transfection, gene silence, Nrf2, shikonin

\section{Introduction}

Lung cancer is seemed as the leading cause of cancer deaths worldwide. Despite the considerable improvement that has been made in the chemotherapy of lung cancer, there is still a problem about intrinsic and acquired resistance to current therapeutic agents [1]. As a majority of lung cancer, nonsmall-cell lung cancers (NSCLC) are generally non-responsive as well as intrinsically resistant to chemotherapy initially. Intrinsic drug resistance happens frequently, but as the chemotherapy becomes more specific and more effective, acquired drug resistance has also become common [1].

Among drug resistance mechanisms, xenobiotics metabolic enzymes in combination with efflux proteins act to detoxify anticancer drugs. However, pro-oxidative drugs induced apoptosis are attenuated by antioxidants via cytoprotective electrophile-responses [2]. It has been shown that the expressions of antioxidants and drug efflux proteins are increased in previous studies on NSCLC [3-5]. Moreover, because the up-regulation of genes are involved in electrophile detoxification, as compared

\footnotetext{
${ }^{1}$ Bo Zhang and Chen Xie contributed equally to this paper.

${ }^{*}$ Corresponding author: Xiaoqin Wang, Key Laboratory of Xinjiang Endemic Phytomedicine Resources, Ministry of Education, Shihezi 832002, China. Tel.: +86-0993-205-7005; Fax: +86-0993-205-7005; E-mail: wangxq5166@sina.com.
}

0959-2989/14/\$27.50 @ 2014 - IOS Press and the authors. 
to normal cells, NSCLC cells exhibit a superior defensive capability against electrophiles [2]. Additionally, NSCLC cells produce abundant multidrug resistance proteins to confer their chemoresistance [6].

The nuclear factor erythroid-2 related factor-2 (Nrf2) is a cap ' $n$ ' collar basic leucine zipper transcription factor which regulates a transcriptional program that maintains cellular redox homeostasis and protects against toxic xenobiotics in normal cells. Keap1 is a cytoplasmic anchor of Nrf2 and sustains steady-state levels of Nrf2 and electrophile-responsive element transcription [7-9]. Electrophiles, oxidants and xenobiotics hinder Nrf2 from the proteasomal degradation mediated by Keap1 and lead to the increasing nuclear accumulation and transcriptional induction of target genes. Transcriptional program regulated by Nrf2 includes a broad spectrum of genes, including genes encoding various antioxidants and enzymes (e.g., the glutathione cycle: $\gamma$-glutamyl-cysteine synthetase modifier subunit, Gclc; $\gamma$-glutamyl-cysteine synthetase catalytic subunit, Gclm; glutathione reductase, GR; glutathione peroxidase, GPX; xenobiotic metabolism cycle: NADP $(\mathrm{H})$ quinone oxidoreductase 1, NQO1; the glutathione-S-transferase family, GSTs; multidrug resistance proteins, MRP). [10-17]. Thus Nrf2 regulated gene products to protect against both oxidants and FAS ligand-induced apoptosis $[18,19]$. Furthermore, down-regulation Nrf2 using anti-sense RNA would be reasonable to improve cell sensitization to apoptosis [19].

As a popular method for exploring gene function, RNA interference (RNAi) suppresses the expression of an interest specific gene in a transformed mammalian cell culture. Knocking-down $\mathrm{Nrf2}$ gene using naked siRNA duplexes in combination with carboplatin significantly inhibits tumor growth in a subcutaneous model of lung cancer [2]. Nrf2 over-expression up-regulated the expression of cytoprotective enzymes, decreased ROS levels, decreased the number of tartrate-resistant acid phosphatase-positive multinucleated cells, reduced marker genes for osteoclast differentiation, and attenuated bone destruction in both in vitro and in vivo models [20]. In fact, the over-expression of Keap1 or the RNAi knockdown of Nrf2 exerted opposite actions [20]. Thus, Nrf2 siRNA inhibitors, in combination with antioxidant depleting drug, may present a novel therapeutic strategy for NSCLC with chemoresistance. Shikonin is a typical anticancer drug used in Traditional Chinese Medicine (TCM) and also seemed as GSH depletor. In reviewing the literatures in NSCLC chemotherapy, no record was found on the association between Nrf2 RNAi and Shikonin. To better understand the chemotherapeutic effects of Nrf2 RNAi plus GSH depletor, the present study aims to determine whether there is the synergistic cytotoxicity effect with Nrf2 gene silence on A549 cells.

\section{Materials and methods}

\subsection{Reagents}

The following items were purchased from the Sigma Chemical Co. (St. Louis, MO, USA): methyl thiazolyl tetrazolium (MTT), 2, 7-dichlorodihydrofluorescein diacetate ( $\mathrm{H}_{2}$ DCFDA), 5chloromethylfluorescein diacetate (CMFDA), and N-acetyl-L-cysteine (NAC). DMEM-F12 media and Fetal bovine serum (FBS) were purchased from the Gibco Life Tech Co. (Shanghai, China). The following items were purchased from the Sangon Biotech Co. (Shanghai,China): total protein extraction kit, TRIzol total RNA Extraction Kit (UNIQ-10), and cDNA synthesis kit. Pst I restriction enzyme and SYBR Premix Ex Taq were purchased from the TaKaRa Biotechnology Co., Ltd (DaLian, China). Lipofectamine 2000 was purchased from Invitrogen Life Tech Co. (Shanghai, China). All other chemicals were of analytical grade and were commercially available. 


\subsection{Cell culture}

The human lung cancer cell line A549 was purchased from the Type Culture Collection of the Chinese Academy of Sciences, Shanghai, China. The cell was maintained at $37^{\circ} \mathrm{C}$ in a $5 \% \mathrm{CO}_{2}$ atmosphere in DMEM-F12 media supplemented with $10 \% \mathrm{FBS}, 100 \mathrm{U} / \mathrm{ml}$ penicillin and $100 \mu \mathrm{g} / \mathrm{ml}$ streptomycin.

\subsection{Plasmid identification}

SureSilencing shRNA Plasmids were purchased from SABioscience (Frederick, MD, USA) (Figure 1A). The SureSilencing shRNA Plasmid insert sequence was used as follows: clone 1: AAA CAA CTC AGC ACC TTA TAT; clone 2: TCA GTC ACT TGT TCC TGA TAT; clone 3: CCA GAA CAC TCA GTG GAA TCT; clone 4: AGG TAA CTG TAG TCC ACA TTT; clone NC: GGA ATC TCA TTC GAT GCA TAC; loop: CTT CCT GTC A. For transformations using DH5 $\alpha$ E.coli Competent Cells, the competent cells and the transformation protocol were prepared according to a modified procedure of Hanahan [21]. Finally, to verify that the purified plasmids contained the shRNA insert, a Pst I restriction enzyme digestion was performed on a small aliquot of each plasmid preparation.

\subsection{Establishment of stable transfected cell lines}

Stable transfected cell lines were developed using SureSilencing shRNA plasmids (Figure 1A), which contain a neomycin resistance marker for the selection of stably transfected cells [22,23]. A549 was transfected with the Nrf2shRNA-1/2/3/4 and shRNA-NC in a six-well plate with the Lipofectamine 2000 transfection reagent. All the procedures were performed by following the reagent supplier's guidance. A selective medium was added containing G418 $(600 \mu \mathrm{g} / \mathrm{mL})$ as the selective antibiotic pressure. Every two to three days, the medium was replaced with fresh medium. In addition, the cells were replated every week. The selection was continued until all of the un-transfected cells had died. The surviving cells were then split into a lower density in a 12-well plate so that each clone stems from 5 to 10 cells. In total, 24 clones were then assessed for the relative knockdown of each specific gene, as well as the related genes, using real-time PCR, and a single clone with the greatest degree of knockdown was selected for subsequent experiments [24].

\subsection{Real Time PCR}

To analyze the expression levels of Nrf2 and its downstream target genes, treated cells were washed with PBS twice and collected. The RNA in each cell sample was extracted using the TRIzol total RNA Extraction Kit (UNIQ-10), then collected to be converted to cDNA using the cDNA synthesis kit (Prime Script RT reagent kit,TaKaRa, China). The Real-time PCR was used to determine the relative gene expression (Rotor Gene Q, Qiagene) with a SYBR Green (Qiagene, USA). The cDNA was submitted to real-time PCR and the sequence of primer pairs are as follows: Nrf2: 5'-ACA CGG TCC ACA GCT CAT C-3'(sense) and 5'-TGT CAA TCA AAT CCA TGT CCT G-3' (anti-sense); NQO1: 5'-AGA GTG GCA TTC TGC ATT TCT G -3'(sense) and 5'-CTG GAG TGT GCC CAA TGC TA 3'(anti-sense); GPX: 5'-GGG GCC TGG TGG TGC TCG GCT -3'(sense) and 5'-CAA TGG TCT GGA AGC GGC GGC-3'(anti-sense); GST: 5'-ATG GGC CTA GAG CTG TTT CT -3'(sense) and 5'-AGC CCA GGT ACT CAT GAA CA-3'(anti-sense) ; HO-1: 5'-GAG TGT AAG GAC CCA TCG GA-3'(sense) and 5'-GCC AGC AAC AAA GTG CAA G-3'(anti-sense); Gclc: 5'- ATG ATG CCA 


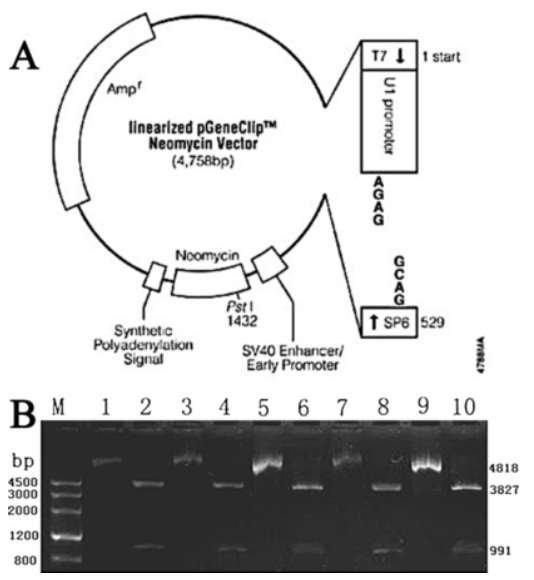

Fig. 1. The pGeneClip ${ }^{\mathrm{TM}}$ Neomycin Vector and plasmid preparation quality control.

A: T7 RNA polymerase transcription initiation site (1), U1 promoter (human, -392 to +1) (46-438), 10bp spacer (439-448), U1 termination sequence (449-465), SP6 RNA polymerase promoter (-17 to +3) (527-546), SP6 RNA polymerase promoter primer binding site (529-547), Binding region of pUC/M13 reverse sequencing primer (564-585), SV40 early enhancer/promoter (798-1216), SV40 minimum origin of replication (1114-1179), Coding region of neomycin phosphotransferase (1251-2045), Synthetic polyA signal (2080-2128), Beta-lactamase (Ampr) coding region (3080-3940), Binding region of pUC/M13 forward sequencing primer (4692-4715), T7 RNA polymerase promoter (-17 to+3)(4742-3).

B: M: 4500 ladder, 1: Nrf2shRNA-1 uncut, 2: Nrf2shRNA-1 Pst I cut, 3: Nrf2shRNA-2 uncut, 4: Nrf2shRNA-2 Pst I cut, 5: Nrf2shRNA-3 uncut, 6: Nrf2shRNA-3 Pst I cut, 7: Nrf2shRNA-4 uncut, 8: Nrf2shRNA-4 Pst I cut, 9: shRNA-NC uncut, 10 : shRNA-NC Pst I cut.

ACG AGT CTG AC-3'(sense) and 5'-CGC CTT TGC AGA TGT CTT TC-3'(anti-sense); Gclm: 5'AGG AGC TTC GGG ACT GTA TT-3'(sense) and 5'-TGG GCT TCA ATG TCA GGG AT-3'(antisense); CAT: 5'-AAC TGG GAT CTT GTG GGA A-3'(sense) and 5'-GAC AGT TCA CAG GTA TCT G-3'(anti-sense); GR: 5'-GAA AAG GCT GTA ATT TTA TTT TCA A-3'(sense) and 5'-ATC AAA AGT CCC TTC CTC TGC-3'(anti-sense); MRP2: 5'-TGA GCA TGC TTC CCA TGA T3'(sense) and 5'-CTT CTC TAG CCG CTC TGT TGG-3'(anti-sense); GAPDH: 5'-CAA GGT CAT CCA TGA CAA CTT TG-3'(sense) and 5'-GTC CAC CAC CCT GTT GCT GTA G-3'(anti-sense) [25-30]. The relative amount of target mRNA was analyzed and calculated by the $2^{-\triangle \Delta \mathrm{Ct}}$ method. The glyceraldehyde-3-phosphate dehydrogenase (GAPDH) was used as an endogenous reference gene for mRNA normalization in each sample.

\subsection{ELISA for Nrf2 present in A549 cells after transfection and in untreated cells}

The total protein was extracted from the cells using the Total protein extraction kit according to the manufacturer's instructions (Sangon Biotech Co., Shanghai, China). The Nrf2 concentrations present in the A549 cells after transfection and present in the untreated cells were measured with a human Nrf2 ELISA kit (Westang Co., Shanghai, China).

\subsection{Determination of intracellular ROS, GSH levels and GSSG/GSH ratio}

The sample cells were washed with PBS and collected to be treated with $\mathrm{H}_{2}$ DCFDA (20 $\left.\mu \mathrm{M}\right)$ (Ex $/ \mathrm{Em}=488 \mathrm{~nm} / 525 \mathrm{~nm})$ or $\mathrm{CMFDA}(5 \mu \mathrm{M})(\mathrm{Ex} / \mathrm{Em}=492 \mathrm{~nm} / 517 \mathrm{~nm})$ at $37^{\circ} \mathrm{C}$ for $30 \mathrm{mins}$. Then, the sample cells were washed twice with PBS, and their relative fluorescence intensity was read by 
spectrophotometry (Varioskan Flash 3001, Thermo, USA). The ROS and GSH levels were calculated as the mean fluorescence intensity per 1,000 non-necrotic cells (MFI), and the measurement of both probes in a single cell was performed using a fluorescence microscope. The GSH/GSSG ratio was determined using a GSH/GSSG assay kit (ATT Bioquest, Inc., USA) according to the manufacturer's instructions.

\subsection{Cell viability assay}

The sample cells were harvested during the exponential phase of growth and then plated into 96well plates. Then, $10 \mu \mathrm{lof} 5 \mathrm{mg} / \mathrm{mL}$ of MTT was added to each well and allowed to react for $4 \mathrm{~h}$. The reaction was halted by adding $150 \mu \mathrm{l}$ of DMSO, and the optical absorbance at $550 \mathrm{~nm}$ was determined by a spectrophotometer. The cell inhibition rate was calculated as $100 \% \times($ control group A values experimental group A values) /control group A values.

\subsection{Statistical analysis}

Each experiment was replicated at least three times. The data obtained from experiments are evaluated by ANOVA and presented as the mean \pm standard deviation of at least three independent experiments. Multiple comparisons were used to identify differences among groups by Student's t-test. Values were considered to be statistically significant at $\mathrm{P}<0.05$.

\section{Results}

\subsection{Plasmid identification}

Five SureSilencing shRNA Plasmids and their Pst I digests were characterized side-by-side on an agarose gel along with a $4500 \mathrm{bp}$ ladder (Figure 1B). The Pst I digests contained the two diagnostic bands indicating that the shRNA insert was present. The uncut plasmid preparations predominantly contained the faster migrating supercoiled form and only very small amounts of the slower migrating nicked plasmids or plasmid concatemers. After being digested with Pst I, all five plasmids generated a diagnostic pair of bands after gel electrophoresis: $3827 \mathrm{bp}$ and $991 \mathrm{bp}$.

\subsection{Generation of A549 cell lines stably expressing Nrf2-shRNA}

To decrease Nrf2 expression level, SureSilencing shRNA plasmids were used and transfected into A549. Compared with the untreated A549 cells (Figure 2A), the transfected cells' morphology had changed to either A549-shRNA-NC or A549-Nrf2shRNA. Furthermore, the cell body becomes long and takes on a stretched appearance (Figures 2B and 2D). However, when undergoing G418 screening (G418: $600 \mu \mathrm{g} / \mathrm{mL}$ ), the transfected cells resembled cluster-like cells (Figure 2C). After screening, two independent clones of Nrf2-shRNA-expressing A549 cells were selected that demonstrated a stable $80 \%$ down-regulation of Nrf2 mRNA (Figure 2E). Furthermore, using ELISA to measure the Nrf2 proteins revealed a similar depressed trend in protein levels. In addition, the Nrf2 expression did not change between the control cells transfected with negative control shRNA and the untransfected cancer cells (Figure 2F). 


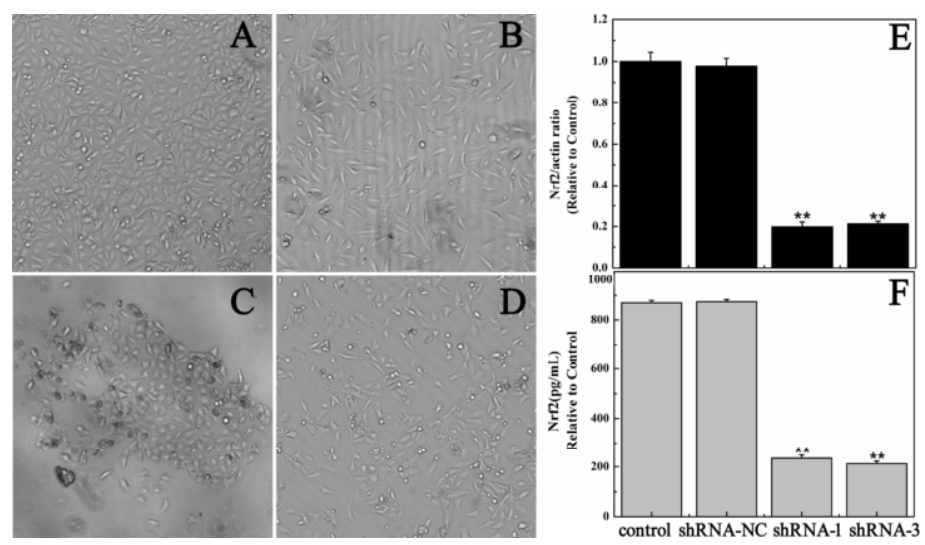

Fig. 2. A549 cell morphological comparison $(\times 100)$ and generation of cell lines stably expressing Nrf2 shRNA A: untreated; B: transfected with shRNA-NC(after G418 screening); C: transfected with Nrf2shRNA (Undergoing G418 screening); D: transfected with Nrf2shRNA (after G418 screening); E: Real time PCR display the expression level of Nrf2 in A549 cells stably expressing Nrf2 shRNA. Total RNA from stable clones harboring Nrf2 shRNA or shRNA-NC were analyzed for expression of Nrf2; F: ELISA detection of Nrf2 in A549 cells stably transfected with shRNA. (**P<0.01 vs. control).

\subsection{Nrf2 gene silence resulting in the down-regulation of xenobiotic metabolism enzymes genes}

As compared with Control and shRNA-NC, the Nrf2 gene silenced two A549 cell clones displayed a decline trend in the expressions of the glutathione pathway genes (Gclm, Gclc, GR, GPX) as indicated in Figure 3. As a result of decreasing Nrf2 levels caused by the shRNA in A549 cells, it was found that other anti-oxidative enzyme genes, such as NQO1, heme oxygenase 1 (HO-1), Catalase (CAT), and GST were down-regulated in present work (Figure 3). Furthermore, the transcription levels of multidrug resistance proteins, like the MRP2, were down-regulated significantly in cells that expressed Nrf2 shRNA. Thus, the down-regulation of Nrf2 profoundly decreased the expression of glutathione pathway genes, antioxidant enzymes and multidrug resistance proteins in A549 cells with the constitutive activation of Nrf2 function.

\subsection{The intracellular redox homeostasis changes in cells stably transfected with Nrf2 shRNA}

As the key result of Nrf2 shRNA silence, the oxidative stress would be increased in response to the global decrease in the expression of the xenobiotic metabolism enzymes genes. To determine the extent of oxidative stress caused by Nrf2 down-regulating, the intracellular redox homeostasis was monitored using both $\mathrm{H}_{2}$ DCFDA and CMFDA fluorescence probes under microscope. The results demonstrated increased fluorescence in A549-Nrf2shRNA (Figures 4A and 4B). A549-Nrf2shRNA cells also demonstrated a significant 25-fold increase in the ROS level. Meanwhile, A549-Nrf2shRNA cells decreased 4-fold in the GSH level (Figures 4A and 4C). Treating these cells with a nonspecific free radical scavenger NAC (2 mM) [2] for 30 mins reduced ROS production and attenuated the mean fluorescent intensity in A549-Nrf2shRNA by 75\%. However, the cells' GSH level did not obviously increase. To precisely define changes of the intracellular redox homeostasis, the ratio of GSH to its oxidized product GSSG was analyzed after selection and NAC treatment. The results show that lowering Nrf2 expression in the A549 cell lines consistently reduced the GSH/GSSG ratio. However, treatment with NAC did not significantly raise the GSH/GSSG ratio (Figure 4D). 

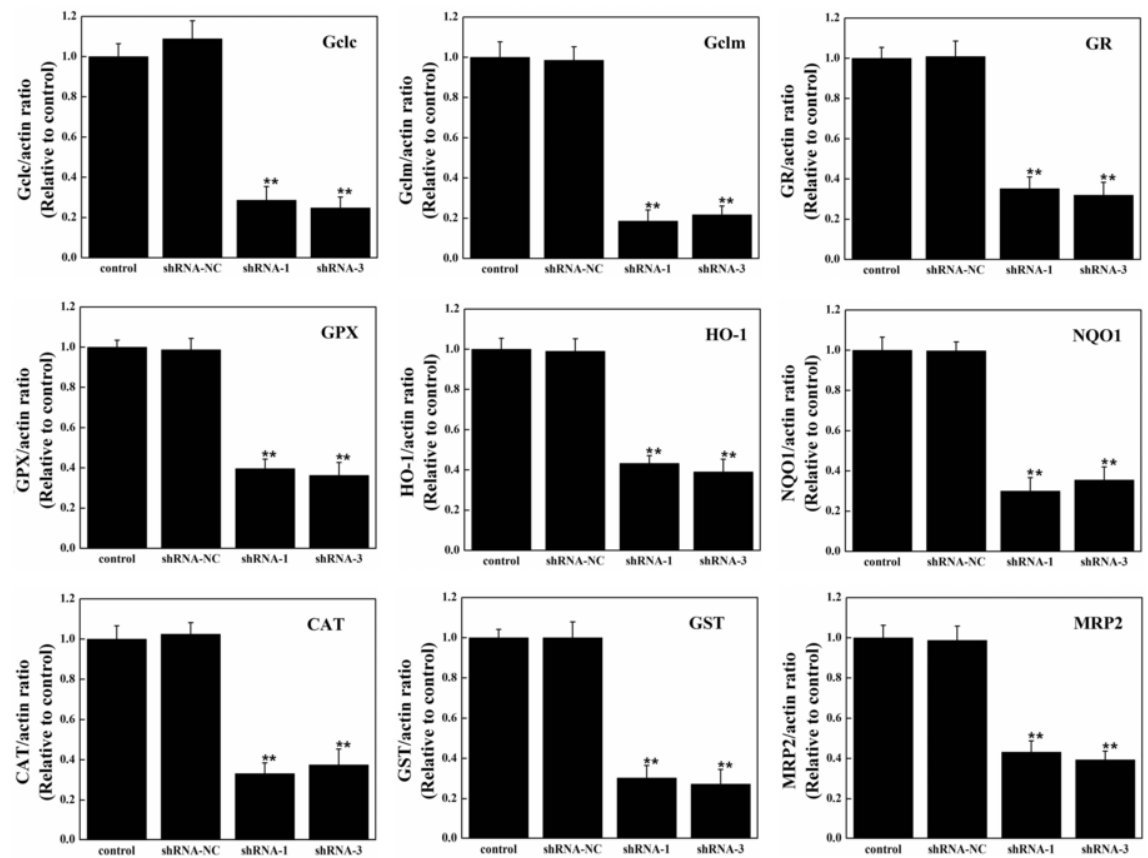

Fig. 3. Key Nrf2 related genes down-regulated in A549-Nrf2shRNA cells

The expressions of several Nrf2 dependent genes were quantified using Real time PCR. The control cells were used as baseline control to calculate the fold changes. All the represented fold change values of Nrf2 shRNA expressing cells are significant compared to the control cells and the cells transfected with shRNA-NC (**P<0.01 vs. control).
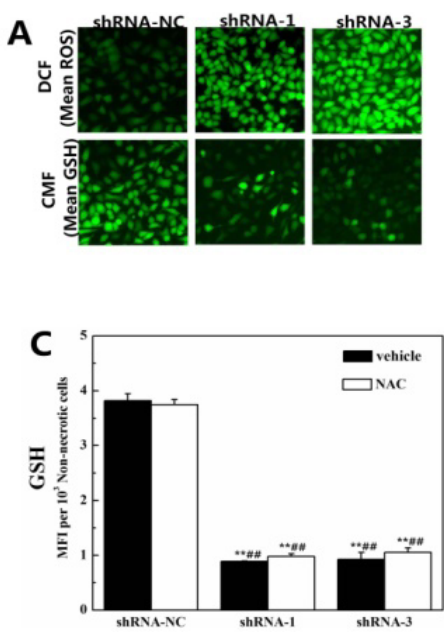
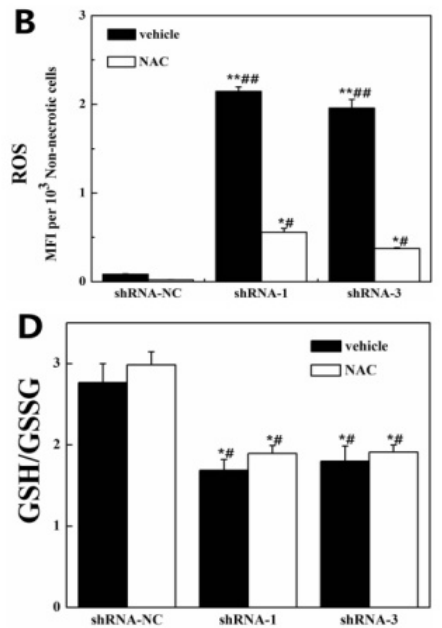

Fig. 4. The changes of intracellular redox homeostasis in cells stably transfected with Nrf2 shRNA

A: The fluorescence probes $\mathrm{H}_{2}$ DCFDA and CMFDA were employed to evaluate the level of the intracellular ROS and GSH in cells stably transfected with Nrf2 shRNA by microscopy. B-C: Pretreatment with NAC (2 mM) and comparison of ROS levels and GSH levels in A549 cells transfected with shRNA-NC, Nrf2shRNA-1 and Nrf2shRNA-3. Cells expressing nontargeting shRNA-NC were used as control. D: The changes of the intracellular GSH/GSSG ratios in cells treated with NAC. $\# \mathrm{P}<0.05, \# \# \mathrm{P}<0.01$ compared to shRNA-NC treated with $\mathrm{NAC},{ }^{*} \mathrm{P}<0.05,{ }^{*} \mathrm{P}<0.01$ compared to shRNA-NC vehicle. 


\subsection{The inhibitory effects of Nrf2 RNAi on A549 cells proliferation}

Since Nrf2 shRNA causes an increase in the ROS level, the inhibition rate of A549 cells that had been stably transfected with shRNA was measured. Untransfected cells were used as the control. The inhibition rate of cells proliferation was analyzed through the MTT assay after screening $2 \mathrm{~d}, 4 \mathrm{~d}$ and $6 \mathrm{~d}$. The inhibition rate was substantially higher in the Nrf2shRNA cells at $2 \mathrm{~d}, 4 \mathrm{~d}$ and $6 \mathrm{~d}$ than in the untransfected cells. The inhibition rate of cells proliferation did not change for the untransfected cells with shRNA-NC cells (Figure 5A).

\subsection{Knockdown of endogenous Nrf2 showing a synergistic cytotoxicity effect with Shikonin on A549 cells}

Shikonin is one of the active components of Lithospermum erythrorhizon Sieb. et Zucc., which chemically reacts with endogenous thiols, including GSH [31]. Since Nrf2 shRNA decreases the expressions of glutathione pathway genes, antioxidants and drug resistance proteins, it was measured that the inhibition rate of Shikonin in A549 cells had been stably transfected with shRNA targeting Nrf2. Untransfected cells were used as the control.

Previous studies have determined that the optimal dose range on cell proliferation inhibition for Shikonin is 2 to $8 \mu \mathrm{M}(0.58$ to $2.31 \mu \mathrm{g} / \mathrm{mL})$ for $24 \mathrm{~h}$ [32]. The experiments in this study, however, used Shikonin within a broad concentration range $(0.8$ to $51.2 \mu \mathrm{g} / \mathrm{mL})$ to extend its cytotoxic efficacy. The inhibition rate of cells proliferation did not change for the untransfected cells with shRNA-NC cells. Either A549-Nrf2shRNA-1 or 3 cell proliferation was inhibited in a dose-dependent manner in comparison to the untransfected cells after $24 \mathrm{~h}$ of Shikonin treatment. As the Shikonin concentration increased from 0.8 to $3.2 \mu \mathrm{g} / \mathrm{mL}$, there was a rapid increase in the inhibition rates, either for the untransfected cells or for the Nrf2shRNA-1/3 cells (Figure 5B). It was found that the decreased Nrf2 level in the A549 cell lines greatly enhanced the cytotoxicity (20-30\%) of Shikonin synergistically, thus resulting in increased cell death when compared to the untransfected cells. In addition,when compared with the control cells that expressed shRNA-NC, A549-Nrf2 shRNA-1/3 cells displayed decreased $\mathrm{IC}_{50}$ score and increased inhibition rate in Shikonin treatments. In summary, the Nrf2 RNAi together with Shikonin treatment showed synergistic cytotoxicity on A549 cells.
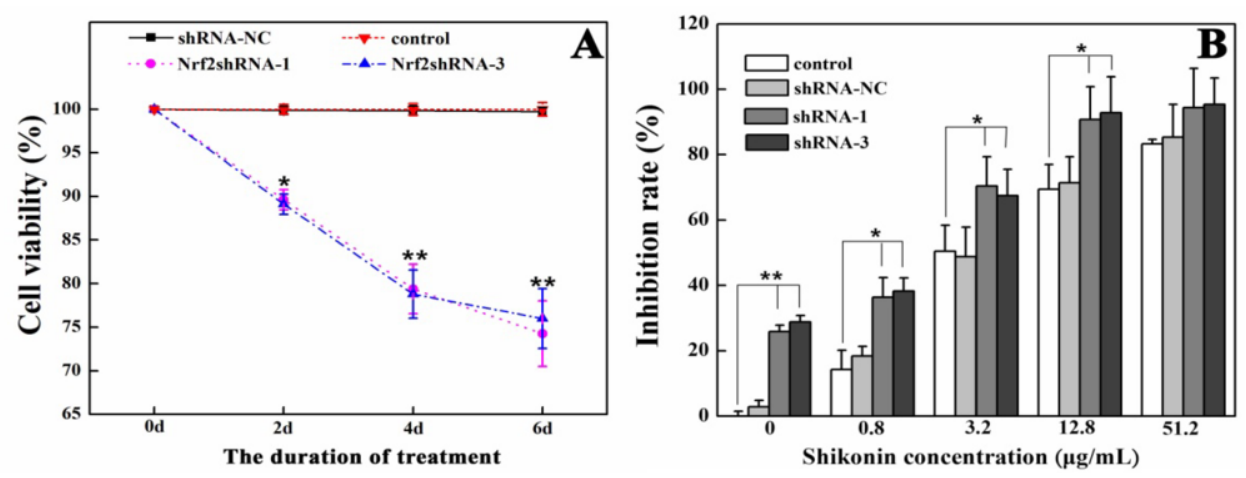

Fig. 5. The inhibitory effect of Shikonin and Nrf2 shRNA on A549

A: The cell viability was determined by the MTT assay after screening $2 \mathrm{~d}, 4 \mathrm{~d}$ and $6 \mathrm{~d}$. B: Shikonin inhibits proliferation in A549, shRNA-NC as well as Nrf2shRNA-1/3 cells. The inhibition rate of cell proliferation was determined by the MTT assay after a $24 \mathrm{~h}$ incubation with Shikonin as indicated concentrations. $* \mathrm{P}<0.05, * * \mathrm{P}<0.01$ compared to control. 


\section{Discussion}

In the present study, it has been demonstrated that NSCLC cells have increased Nrf2 activity due to Keap1 genetic somatic mutations [33-35]. If Keap1 lost activity, which leads to the constitutive activation of Nrf2 in NSCLC cells, up-regulates the expressions of drug detoxification enzymes and efflux proteins $[33,35]$. To understand the functional significance of gaining Nrf2 function in NSCLC, then an RNAi approach was used and a lung cancer A549 cell line was selected that has been previously reported to achieve the loss of Keap1 activity [35].

The SureSilencing shRNA plasmids are employed in present study according to an experimentally verified algorithm. These constructs specifically down-regulate the expression of specific genes by RNAi and allow for the enrichment or selection of transfected cells [22,23]. Under the control of the U1 promoter and the Neomycin gene, the short hairpin RNA (shRNA) is expressed by using the vector. All three plasmid types were detected by gel electrophoresis and generated a diagnostic pair of bands. The Pst I digests contained the two diagnostic bands, which indicated that the shRNA insert was present. The uncut plasmid preparations predominantly contained the faster migrating supercoiled form and only very small amounts of the slower migrating nicked plasmid or plasmid concatamers.

Previous study had reported a rapid enhancement in ROS production and, conversely, a depletion in GSH as a preliminary effect of HL-60 cell differentiation in Shikonin treatments [36], which agrees well with previously published pro-apoptotic reports [19,37,38]. Based on the present study, it should be determined whether there is a regulatory role of the Nrf2 pathway to replenish GSH and other antioxidants to scavenge ROS and other electrophilic molecules [39,40]. In constructing the GSH system, GCL is a key enzyme to perform GSH synthesis as GR. GCL composed of a catalytic subunit (Gclc) and a modulatory subunit (Gclm) [28]. GPX was responsible for GSH oxidation to GSSG by $\mathrm{H}_{2} \mathrm{O}_{2}$ [41]. NQO1 was responsible for reduction of quinones to hydroquinones by consuming a reducing matter as NADPH [42]. Nrf2 RNAi in the A549 cells resulted in a marked decrease in the expression of genes that constitute the glutathione cycle (GSH bio-synthesizing enzymes, GPX, and GR, antioxidants (HO-1, NQO1, and CAT), and drug resistance proteins (MRP2) [14,43]. In corroboration with the GR, GPX and GST gene expression as well as the total GSH levels were significantly reduced in Nrf2 shRNAi A549 cells and in comparison with the shRNA-NC control cells. Thus, Nrf2 gene expression down-regulation profoundly decreased the expression of key antioxidative enzyme genes, glutathione pathway genes and multidrug resistance proteins in NSCLC cells with a constitutive activation of Nrf2 function.

The down-regulation of the Nrf2 pathway caused a dramatic ROS accumulation and GSH depletion in the A549-Nrf2shRNA-1/3 cells; simultaneously, the redox homeostasis in the cells was shifted towards oxidation. Treating these cells with GSH precursor NAC reduced ROS production in the A549-Nrf2shRNA-1/3 cells. Further, during chemotherapy by platinum, cancer cells could be protected by NAC not only through antioxidant but also through the covalent binding of the molecule to the platinum, thus producing an inactive complex [44-46]. However, the NAC pretreatment did not obviously restore the GSH levels in Nrf2 knockdown cells, which suggests that the gene expressions in the GSH biosynthesis that is positively regulated by Nrf2 were also inhibited by RNAi.

Chemotherapy is one of the primary methods used for cancer treatments. The production of reactive oxygen species (ROS) plays an important role in the apoptosis by commonly used chemotherapy agents, such as cisplatin, bleomycin, paclitaxel, adriamycin and etoposide [47,48]. However, developing a resistance to anticancer agents limits its therapeutic use for cancer treatments. Drugresistant cancer cells may be involved in several different mechanisms, such as increased cellular detoxification and antioxidant systems [49-51]. In addition, GSH is a major endogenous antioxidant 
that contributes to rescuing cells from apoptosis. Previous studies have suggested that an elevated cellular GSH level caused decreased responses to chemotherapeutic agents [51,52]. In human lung cancer, the siRNA-mediated repression of self-defense genes and of cellular GSH pool led to an inhibition of cell growth and a sensitivity to anticancer agents [53]. Similar to our study, cell viability was substantially lower in the Nrf2shRNA-1/3 cells as compared to the untransfected cells.

In TCM, a famous "heat clearing and blood cooling" herb "shikon" grows widely in Xin Jiang region of China. Additionally, the root extracts of shikon have been used in TCM for many years as a form of cancer treatment [54]. Shikonin, the main naphthoquinone compound of the shikon root, was recently demonstrated as having pro-apoptotic effects and pro-oxidative activities on various cells [55]. Although ROS production from mitochondria was seemed to trigger apoptosis by Shikonin and other anticancer drugs [56], in Gao et al.'s study, their results indicate that Shikonin directly reacts with GSH and other oxidative stress-relevant compounds in the lysate of HL-60 cells [31]. In our study, a combination of Nrf2 shRNA with Shikonin led to an increase in the inhibition rate as compared to the control. Changes in cell viability/inhibition rates were attributed to the decreased GSH levels synergistically caused by Nrf2 shRNA silences as well as Shikonin treatments.

The constitutive activation of $\mathrm{Nrf} 2$ mediates important defensive role in electrophile detoxification and drug resistance in NSCLC cells at transcriptional level. The application of biochemical RNAi silencing of the Nrf2 gene and chemotherapeutical GSH depletor are promising strategies to inhibit cell proliferation in lung cancer cells.

\section{Acknowledgment}

This study was supported by the key areas of Science and Technology Project (NO. 2014BA029) of the Xinjiang production \& Construction Corps and the funds for Distinguished Young Scientists of Shihezi University to Bo Zhang (2013ZRKXJQ04).

\section{References}

[1] A. Nadkar, C. Pungaliya, K. Drake, E. Zajac, S.S Singhal and S. Awasthi, Therapeutic resistance in lung cancer, Expert Opin. Drug Metab. Toxicol. 2 (2006), 753-777.

[2] A. Singh, S. Boldin-Adamsky, R.K. Thimmulappa, S.K. Rath, H. Ashush, J. Coulter et al., RNAi mediated silencing of Nrf2 gene expression in non-small cell lung cancer inhibits tumor growth and increases efficacy of chemotherapy, Cancer Res. 68 (2008), 7975-7984.

[3] Y. Soini, U. Napankangas, K. Jarvinen, R. Kaarteenaho-Wiik, P. Paakko and V.L. Kinnula, Expression of gammaglutamyl cysteine synthetase in nonsmall cell lung carcinoma, Cancer 92 (2001), 2911-2919.

[4] K.D. Tew, Glutathione-associated enzymes in anticancer drug resistance, Cancer Res. 54 (1994), 4313-4320.

[5] P. Yang, J.O. Ebbert, Z. Sun and R.M. Weinshilboum, Role of the glutathione metabolic pathway in lung cancer treatment and prognosis: A review, J. Clin. Oncol. 24 (2006), 1761-1769.

[6] D. Trachootham, Y. Zhou, H. Zhang, Y. Demizu, Z. Chen, H. Pelicano et al., Selective killing of oncogenically transformed cells through a ROS-mediated mechanism by beta-phenylethyl isothiocyanate, Cancer Cell 10 (2006), 241252.

[7] A. Kobayashi, M.I. Kang, H. Okawa, M. Ohtsuji, Y. Zenke, T. Chiba et al., Oxidative stress sensor Keap1 functions as an adaptor for Cul3-based E3 ligase to regulate proteasomal degradation of Nrf2, Mol. Cell Biol. 24 (2004), 7130-7139.

[8] D.D. Zhang, S.C. Lo, J.V. Cross, D.J. Templeton and M. Hannink, Keap1 is a redox-regulated substrate adaptor protein for a Cul3-dependent ubiquitin ligase complex, Mol. Cell Biol. 24 (2004), 10941-10953.

[9] T.W. Kensler, N. Wakabayashi and S. Biswal, Cell survival responses to environmental stresses via the Keap1-Nrf2ARE pathway, Annu. Rev. Pharmacol. Toxicol. 47 (2007), 89-116. 
[10] A.Hayashi, H. Suzuki, K. Itoh, M. Yamamoto and Y. Sugiyama, Transcription factor Nrf2 is required for the constitutive and inducible expression of multidrug resistance-associated protein 1 in mouse embryo fibroblasts, Biochem. Biophys. Res. Commun. 310 (2003), 824-829.

[11] T. Nguyen, P.J. Sherratt and C.B. Pickett, Regulatory mechanisms controlling gene expression mediated by the antioxidant response element, Annu. Rev. Pharmacol. Toxicol. 43 (2003), 233-260.

[12] V. Vollrath, A.M. Wielandt, M. Iruretagoyena and J. Chianale, Role of Nrf2 in the regulation of the Mrp2 (ABCC2) gene, Biochem. J. 395 (2006), 599-609.

[13] Y.J. Kim, J.Y. Ahn, P. Liang, C. Ip, Y. Zhang and Y.M. Park, Human prx1 gene is a target of Nrf2 and is up-regulated by hypoxia/reoxygenation: implication to tumor biology, Cancer Res. 67 (2007), 546-554.

[14] T.D. Lee, H. Yang, J. Whang and S.C. Lu, Cloning and characterization of the human glutathione synthetase 5'flanking region, Biochem. J. 390 (2005), 521-528.

[15] T. Rangasamy, C.Y. Cho, R.K. Thimmulappa, L. Zhen, S.S. Srisuma, T.W. Kensler, M. Yamamoto et al., Genetic ablation of Nrf2 enhances susceptibility to cigarette smoke-induced emphysema in mice, J. Clin. Invest. 114 (2004), $1248-1259$.

[16] T. Rangasamy, J. Guo, W.A. Mitzner, J. Roman, A. Singh, A.D. Fryer et al., Disruption of Nrf2 enhances susceptibility to severe airway inflammation and asthma in mice, J. Exp. Med. 202 (2005), 47-59.

[17] R.K. Thimmulappa, C. Scollick, K. Traore, M. Yates, M.A. Trush, K.T. Liby et al., Nrf2-dependent protection from LPS induced inflammatory response and mortality by CDDO-Imidazolide, Biochem. Biophys. Res. Commun. 351 (2006), 883-889.

[18] K.U. Kotlo, F. Yehiely, E. Efimova, H. Harasty, B. Hesabi, K. Shchors et al., Nrf2 is an inhibitor of the Fas pathway as identified by Achilles' Heel Method, a New function-based approach to gene identification in human cells, Oncogene 22 (2003), 797-806.

[19] N. Morito, K. Yoh, K. Itoh, A. Hirayama, A. Koyama, M. Yamamoto et al., Nrf2 regulates the sensitivity of death receptor signals by affecting intracellular glutathione levels, Oncogene 22 (2003), 9275-9281.

[20] H. Kanzaki, F. Shinohara, M. Kajiya and T. Kodama, The Keap1/Nrf2 protein axis plays a role in osteoclast differentiation by regulating intracellular reactive oxygen species signaling, J. Biol. Chem. 288 (2013), 23009-23020.

[21] D. Hanahan, DNA cloning, D.M. Glover, ed., ORL Press, Oxford, Washington DC, 1985, vol. 1, pp. 109-135.

[22] G. Frampton, M. Coufal, H. Li, J. Ramirez and S. DeMorrow, Opposing actions of endocannabinoids on cholangiocarcinoma growth is via the differential activation of Notch signaling, Exp. Cell Res. 316 (2010), 1465-1478.

[23] L. Huang, J.C. Ramirez, G.A. Frampton, L.E. Golden, M.A. Quinn, H.Y. Pae et al., Anandamide exerts its antiproliferative actions on cholangiocarcinoma by activation of the GPR55 receptor, Lab Invest. 91 (2010), 1007-1017.

[24] S. DeMorrow, H. Francis, E. Gaudio, J. Venter, A. Franchitto, S. Kopriva et al., The endocannabinoid anandamide inhibits cholangiocarcinoma growth via activation of the noncanonical Wnt signaling pathway, Am. J. Physiol. Gastrointest. Liver Physiol. 295 (2008), 1150-1158.

[25] N. Wang, L.W. Wang, B.D. Gou, T.L. Zhang and K. Wang, Realgar-induced differentiation is associated with MAPK pathways in HL-60 cells, Cell Biol. Int. 32 (2008), 1497-1505.

[26] S. Caprodossi, M. Pedinotti, C. Amantini, G. Santoni, S. Minucci, P.G. Pelicci et al., Differentiation response of acute promyelocytic leukemia cells and PML/RAR $\alpha$ leukemogenic activity studies by real-time RT-PCR, Mol. Biotechnol. 30 (2005), 231-238.

[27] T.W. Fischer, M.A. Zmijewski, B. Zbytek, T.W. Sweatman, R.M. Slominski, J. Wortsman et al., Oncostatic effects of the indole melatonin and expression of its cytosolic and nuclear receptors in cultured human melanoma cell lines, Int. J. Oncol. 29 (2006), 665-672.

[28] H.R. Lee, J.M. Cho, D.H. Shin, C.S. Yong, H.G. Choi, N. Wakabayashi et al., Adaptive response to GSH depletion and resistance to L-buthionine-(S,R)-sulfoximine: involvement of Nrf2 activation, Mol. Cell Biochem. 318 (2008), $23-31$.

[29] M.G. Baigi, L. Brault, A. Néguesque, M. Beley, R.E. Hilali, F. Gaüzère et al., Apoptosis/necrosis switch in two different cancer cell lines: Influence of benzoquinone and hydrogen peroxide-induced oxidative stress intensity, and glutathione, Toxicol. in Vitro. 22 (2008), 1547-1554.

[30] T. Amalraj, R. Jessica, K.K. Naveen, W.F. James and A. Sudhakar, Novel function of transcription factor Nrf2 as an inhibitor of RON tyrosine kinase receptor mediated cancer cell invasion, J. Biol. Chem. 28 (2011), 31-35.

[31] D. Gao, M. Hiromura, H. Yasui and H. Sakurai, Direct reaction between shikonin and thiols induces apoptosis in HL60 cells, Biol. Pharm. Bull. 25 (2002), 827-832.

[32] H. Wang, C. Wu, S. Wan, H. Zhang, S. Zhou and G. Liu, Shikonin attenuates lung cancer cell adhesion to extracellular matrix and metastasis by inhibiting integrin $\beta 1$ expression and the ERK1/2 signaling pathway, Toxicology 308 (2013), 104-112.

[33] B. Padmanabhan, K.I. Tong, T. Ohta, Y. Nakamura, M. Scharlock, M. Ohtsuji et al., Structural basis for defects of Keap1 activity provoked by its point mutations in lung cancer, Mol. Cell 21 (2006), 689-700. 
[34] T. Sjoblom, S. Jones, L.D. Wood, D.W. Parsons, J. Lin, T.D. Barber et al., The consensus coding sequences of human breast and colorectal cancers, Science 314 (2006), 268-274.

[35] T. Kurosu, T. Fukuda, T. Miki and O. Miura, BCL6 overexpression prevents increase in reactive oxygen species and inhibits apoptosis induced by chemotherapeutic reagents in B-cell lymphoma cells, Oncogene 22 (2003), 4459-4468.

[36] B. Zhang, N. Chen, H. Chen, Z. Wang and Q. Zheng, The critical role of redox homeostasis in Shikonin-induced HL-60 cell differentiation via unique modulation of the Nrf2/ARE Pathway, Oxid. Med. Cell Longev. 2012 (2012), 781516.

[37] W. Han, J. Xie, L. Li, Z. Liu and X. Hu, Necrostatin-1 reverts shikonin-induced necroptosis to apoptosis, Apoptosis 14 (2009), 674-686.

[38] W. Han, J. Xie, Y. Fang, Z. Wang and H. Pan, Nec-1 Enhances shikonin-induced apoptosis in leukemia cells by inhibition of RIP-1 and ERK1/2, Int. J. Mol. Sci. 13 (2012), 7212-7225.

[39] S.M. Krance, P.C. Keng, J.P. Palis and N. Ballatori, Transient glutathione depletion determines terminal differentiation in HL-60 cells, Oxid. Med. Cell Longev. 3 (2010), 53-60.

[40] I. Bobilev, V. Novik, I. Levi, O. Shpilberg, J. Levy, Y. Sharoni et al., The Nrf2 transcription factor is a positive regulator of myeloid differentiation of acute myeloid leukemia cells, Cancer Biol. Ther. 11 (2011), 317-329.

[41] F.Q. Schafer and G.R. Buettner, Redox environment of the cell as viewed through the redox state of the glutathione disulfide/glutathione couple, Free Radic. Biol. Med. 30 (2001), 1191-1212.

[42] A.T. Dinkova-Kostova and P. Talalay, NAD (P) H: quinone acceptor oxidoreductase 1 (NQO1), a multifunctional antioxidant enzyme and exceptionally versatile cytoprotector, Arch. Biochem. Biophys. 501 (2010), 116-123.

[43] T. Ohta, K. Iijima, M. Miyamoto, I. Nakahara, H. Tanaka, M. Ohtsuji et al., Loss of keap1 function activates Nrf2 and provides advantages for lung cancer cell growth, Cancer Res. 68 (2008), 1303-1309.

[44] D.T. Dickey, Y.J. Wu, L.L. Muldoon and E.A. Neuwelt, Protection against cisplatin-induced toxicities by Nacetylcysteine and sodium thiosulfate as assessed at the molecular, cellular, and in vivo levels, J. Pharmacol. Exp. Ther. 314 (2005), 1052-1058.

[45] M.A. Fuertes, J. Castilla, C. Alonso and J.M. Perez, Novel concepts in the development of platinum antitumor drugs, Curr. Med. Chem. Anticancer Agents 2 (2002), 539-551.

[46] E.A. Neuwelt, M.A. Pagel, B.P. Hasler, T.G. Deloughery and L.L. Muldoon, Therapeutic efficacy of aortic administration of $\mathrm{N}$-acetylcysteine as a chemoprotectant against bone marrow toxicity after intracarotid administration of alkylators, with or without glutathione depletion in a rat Model, Cancer Res. 61 (2001), 7868-7874.

[47] H. Masuda, T. Tanaka and U. Takahama, Cisplatin generates superoxide anion by interaction with DNA in a cell-free system, Biochem. Biophys. Res. Commun. 203 (1994), 1175-1180.

[48] D. Trachootham, Y. Zhou, H. Zhang, Y. Demizu, Z. Chen, H. Pelicano et al., Selective killing of oncogenically transformed cells through a ROS-mediated mechanism by beta-phenylethyl isothiocyanate, Cancer Cell 10 (2006), 241252.

[49] S. Homma, Y. Ishii, Y. Morishima, T. Yamadori, Y. Matsuno, N. Haraguchi et al., Nrf2 enhances cell proliferation and resistance to anticancer drugs in human lung cancer, Clin. Cancer Res. 15 (2009), 3423-3432.

[50] T. Jiang, N. Chen, F. Zhao, X.J. Wang, B. Kong, W. Zheng et al., High levels of Nrf2 determine chemoresistance in type II endometrial cancer, Cancer Res. 70 (2010), 5486-5496.

[51] G.S. Shim, S. Manandhar, D.H. Shin, T.H. Kim and M.K. Kwak, Acquisition of doxorubicin resistance in ovarian carcinoma cells accompanies activation of the NRF2 pathway, Free Radic. Biol. 47 (2010), 1619-1631.

[52] K. Zhang, E.B. Yang, K.P. Wong and P. Mack, GSH-related enzymes and GS-X pump in relation to sensitivity of human tumor cell lines to chlorambucil and adriamycin, Int. J. Oncol. 14 (1999), 861-867.

[53] P. Yang, J.O. Ebbert, Z. Sun and R.M. Weinshilboum, Role of the glutathione metabolic pathway in lung cancer treatment and prognosis: a review, J. Clin. Oncol. 24 (2006), 1761-1769.

[54] V.P. Papageorgiou, A.N. Assimopoulou, E.A. Couladouros, D. Hepworth and K.C. Nicolaou, The chemistry and biology of alkannin, shikonin, and related naphthazarin natural products, Angew. Chem. Int. Ed. 38 (1999), 270-301.

[55] X. Mao, C.R. Yu, W.H. Li and W.X. Li, Induction of apoptosis by shikonin through a ROS/JNK-mediated process in Bcr/Abl-positive chronic myelogenous leukemia (CML) cells, Cell Res. 18 (2008), 879-888.

[56] Y.B. Ji, C.F. Ji and L. Yue, Human gastric cancer cell line SGC-7901 apoptosis induced by SFPS-B2 via a mitochondrial-mediated pathway, Biomed. Mater. Eng. 24 (2014), 130914. 